\title{
Evaluación de la exploración de los pacientes diabéticos y de la educación diabetológica en los centros del Servicio Andaluz de Salud de Malaga (España)
}

\author{
Evaluation of the assessment of diabetic patients and the diabetology \\ education at the Servicio Andalud de Salud in Malaga (Spain)
}

\author{
José Luis Ledesma Vargas ${ }^{1}$, Isabel Andujar Lázaro ${ }^{2}$, Joaquín Páez Moguer ${ }^{3}$ \\ ${ }^{1}$ Profesor Asociado de Podología. Universidad de Málaga. Diplomado en Podología. Diplomado en enfermería. \\ Subinspector Delegación Salud Málaga \\ josledvar@uma.es \\ ${ }^{2}$ Epidemióloga. Distrito Axarquía. Servicio Andaluz de Salud \\ isandujar5@gmail.com \\ ${ }^{3}$ Profesor Asociado de Podología. Universidad de Málaga. Diplomado en Podología \\ joapaemog@uma.es
}

Correspondencia:

Isabel Andújar Lázaro

C/ Martínez Maldonado 35, $3^{\circ} \mathrm{J}$

E-29007 Málaga

Fecha de recepción: 14 de octubre de 2009

Fecha de aceptación: 1 de diciembre 2009

Los autores declaran no tener ningún tipo de interés económico o comercial.

\section{RESUMEN}

Cada vez es mayor el $\mathrm{n}^{\mathrm{o}}$ de pacientes diabéticos en el mundo, según estimaciones de la O.M.S. tenemos 180 millones de personas en el mundo que la padecen. En España asumimos en torno a un 6,5\% de la población afectada.

El pie diabético es una de las complicaciones que con mayor frecuencia sufren los pacientes diabéticos. La angiopatía y neuropatía que conlleva la diabetes, es más acusada en las zonas distales de nuestro cuerpo en especial el pie. El aumento de la prevalencia de diabéticos provoca que exista también un aumento en el número de casos de pie diabético.

El Plan integral de Diabetes de Andalucía 2003-2007 señala lo siguiente: "el pie diabético es fundamentalmente un problema de prevención".

En el presente trabajo se analiza la exploración del pie diabético y la educación diabetológica en los centros del S.A.S. de la provincia de Málaga.

Se observan deficiencias tanto en equipamiento material como humano.

Por último queremos hacer hincapié en la necesidad de la inclusión del podólogo en los programas de salud del pie diabético. El pie diabético es una patología grave, compleja, de un altísimo coste económico y humano, y que requiere un abordaje multidisciplinar.

Palabras clave: Pie Diabético; Educación Diabetes.

\begin{abstract}
ABSTRAST
Every time $\mathrm{n}^{\mathrm{o}}$ of diabetic patients in the world is greater, according to estimations of the O.M.S. we have 180 million people in the world who suffer it. In Spain we have around a $6.5 \%$ of the affected population. The diabetic foot is one of the complications that most frequently undergo the diabetic patients. The angiopathy and neuropathy that the diabetes entails, are more accusing in the distad zones of our special body in the foot. The increase of the prevalence of diabetics causes that an increase in $n^{\circ}$ of cases of diabetic foot also exists. In
\end{abstract}


Andalusia one settled down in the integral Plan of Diabetes in Andalusia 2003-2007 and as it indicates the own plan: "the diabetic foot is fundamental a prevention problem". In the present work we expose, as it is carried out the exploration of the diabetic foot and the diabetes education within the medicine consultations of the S.A.S. in the province of Malaga. We have observed that exists deficiencies in the material and human resources as much. Finally we want to insist on the necessity of the inclusion of the podiatry in the programs of health of the diabetic foot. The diabetic foot is a serious, complex pathology, of a highest economic and human cost, and that requires a boarding to multidiscipline.

Key words: Diabetic Foot; Diabetes Education.

Sumario: 1. Introducción. 2. Material y Método. 3. Resultados. 4. Discusión. Bibliografía.

Referencia bibliográfica: Ledesma Vargas, JL., Andujar Lázaro, I., Páez Moguer, J. Evaluación de la exploración de los pacientes diabéticos y de la educación diabetológica en los centros del Servicio Andaluz de Salud de Malaga (España). Rev. Int. Cienc. Podol. 2012; 6(1): 51-57. 


\section{INTRODUCCIÓN}

La diabetes es una enfermedad con elevada morbilidad y una importante causa de mortalidad prematura. Según las estimaciones de la Organización Mundial de la Salud, más de 180 millones de personas en el mundo tienen diabetes, siendo probable que se duplique este dato antes del 2030. Se estima que una de cada 20 muertes en el mundo es atribuible a la esta enfermedad, de tal manera que la proporción es de una de cada 10 muertes en la población entre los 35 y 64 años de edad. En el año 2005, 1,1 millones de personas fallecieron a causa de la diabetes, la mitad de las muertes afectó a menores de 70 años, y más de la mitad $(55 \%)$ a mujeres ${ }^{(1)}$.

En España, en 2004, la Diabetes Mellitus causó 9.966 muertes, de las que 3.924 fueron varones y 6.042 mujeres. A estos datos habría que añadir un porcentaje desconocido de muertes por enfermedad cardiovascular que puede ser directamente atribuible a esta enfermedad ${ }^{(1)}$.

La prevalencia estimada de diabetes en nuestro país se sitúa en torno a un 6,5\% para la población entre los 30 y 65 años, oscilando en diversos estudios entre el 6 y el $12 \%{ }^{(1)}$.

En Andalucía existen escasos estudios que detallen la prevalencia de la diabetes. Sí existen estudios sobre la prevalencia de diabetes tratadas con fármacos en Andalucía, pero esta información sólo nos proporciona datos sobre la diabetes tratada, no de la diabetes real existente ${ }^{(2,3)}$.

La edad avanzada, la obesidad, la presencia de historia familiar de diabetes, el sedentarismo, el nivel socioeconómico bajo, la tolerancia alterada a la glucosa, la hipertensión arterial y la hiperlipemia son factores de riesgo que se asocian con el desarrollo de diabetes. El aumento en la prevalencia de la obesidad y del consumo de tabaco está contribuyendo a aumentar la incidencia de la enfermedad ${ }^{(2)}$.

Distintos estudios indican que el control estricto de los factores de riesgo cardiovascular en los diabéticos tipo 2 puede disminuir en un $50 \%$ la aparición de complicaciones micro y macrovasculares y reducir el riesgo de mortalidad. Asimismo, mediante la promoción de estilos y hábitos de vida saludables, la actividad física y de una dieta saludable entre otras me- didas, se puede evitar una proporción elevada de casos de diabetes tipo $2^{(1)}$.

En cuanto al coste económico, se calcula que la población con diabetes puede consumir entre un 4 y un $14 \%$ del gasto sanitario global de los países de nuestro entorno, siendo en España pocos los estudios relacionados con este fin ${ }^{(4)}$

Las úlceras y amputaciones son las principales causas de morbilidad, discapacidad y coste en los pacientes diabéticos. Se estima que en torno a un $15 \%$ de los pacientes con diabetes desarrollarán en algún momento de sus vidas una úlcera en un miembro inferior y que entre un $14-20 \%$ de los pacientes diabéticos con úlceras requerirán una amputación, atribuyéndose al pie diabético entre el $25-50 \%$ de los costos de hospitalización de la diabetes ${ }^{(5)}$.

La prevalencia de pie diabético oscila entre el 8-13\%. Las lesiones del pie diabético suponen entre un $20-50 \%$ de los ingresos hospitalarios del paciente con diabetes. El coste medio de la asistencia al pie diabético, se calcula que es de unos 27.000€ $€^{2(5)}$.

Por todo lo anterior, la prevención de estas lesiones cobra una especial importancia, y se realiza fundamentalmente a través de la identificación de paciente con pies de riesgo de ulceración.

La detección precoz de las lesiones requiere una exploración cuidadosa de la sensibilidad en sus distintas vertientes: táctil, dolorosa, térmica y vibrátil, en la que el empleo de algunas técnicas como la aplicación de monofilamentos de Semmes-Weinstein se considera de elección para la detección del pié de riesgo. Existen diversos tipos de monofilamentos, el más utilizado para el pie diabético es el monofilamento de $5,07 \mathrm{~mm}$ que al aplicarlo e incurvarse produce $10 \mathrm{~g}$ de presión $^{(7)}$.

Con la percepción o no de esta presión comprobamos el umbral de protección, siendo este el punto crítico en el que el sistema sensitivo no puede proteger al tejido blando Por tanto el paciente que carezca de este umbral de percepción será más susceptible ante determinadas agresiones externas debido a que no puede percibirlas.

Esta técnica de exploración mediante el Monofilamento de Semmes-Weinstein es considerada por algunos expertos como de elección 
para la detección precoz del pie de riesgo, al ser simple y barata. Combina 3-4 puntos, teniendo razonable sensibilidad y especificidad en relación al tiempo disponible en Atención Primaria $^{(7)}$.

El riesgo de ulceración y amputación puede reducirse a través de programas estructurados de educación en el cuidado de los pies. Asimismo, existe evidencia sobre la eficacia del cribado y cuidado de los pies de riesgo en la reducción de úlceras y amputaciones.

El Plan Integral de diabetes en Andalucía contempla una actuación integral sobre esta enfermedad, y recoge como elemento clave la Gestión por Procesos Asistenciales Integrados con el objetivo de mejorar los resultados en salud y la calidad de vida de las personas afectadas $^{(2,6)}$. El Plan de Inspección de 2008, incluye un programa de evaluación de pacientes diabéticos y de la evaluación diabetológica, con el fin de evaluar determinadas actividades del Plan Integral de Diabetes (2003-2007).

Los objetivos de presente trabajo son verificar el cumplimiento de algunas de las actividades propuestas en el Plan Integral de Diabetes en Andalucía tales como la disponibilidad del material de exploración general y de los pies en pacientes con diabetes, la utilización de dicho material y la existencia de programas de educación diabetológica en cada centro evaluado. Evaluar las normas de calidad incluidas en los procesos Diabetes I y II

\section{MATERIAL Y MÉTODO}

La información ha sido recogida mediante entrevista personal con el responsable de cada centro, a través de dos cuestionarios elaborado al efecto por la Subdirección de Inspección de Servicios Sanitarios de la Consejería de salud de la Junta de Andalucía.

La elección de los centros a evaluar, se realizó mediante muestreo aleatorio estratificado proporcional al total de centros existentes incluyendo un total de 35 centros de Salud y hospitales de Málaga y provincia . Para evaluar las normas de calidad del proceso asistencial y la exploración se realizó un muestreo simple del total de historias clínicas en las que constaba el diagnóstico de diabetes tipo I o II. La información fue obtenida durante el primer semestre de 2008.

\section{RESULTADOS}

En los centros de salud, se han evaluado un total de 192 consultas, tanto de medicina de familia como de enfermería y pediatría.

En cuanto a disponibilidad de material, el 96\% de las consultas disponen de monofilamentos de Semmens-Wenstein para la exploración de la sensibilidad a efectos de la detección de neuropatía diabética y el $100 \%$ tienen esfigmomanómetros de tamaño normal, estando calibrados el $72.4 \%$.

El $84.7 \%$ de las consultas de medicina de familia disponen de material mínimo para la exploración general de pacientes diabéticos, mientras que este porcentaje es considerablemente menor $(15.4 \%)$ en las consultas de pediatría (Tabla 1).

Con respecto a la disponibilidad de material mínimo para le exploración de los pies de los diabéticos el 93\% de las consulta de medicina general lo tienen, frente al $7 \%$ de las consultas de pediatría (Tabla 2).

\begin{tabular}{lrrrrr}
\hline $\begin{array}{l}\text { Disponibilidad de material mínimo para la } \\
\text { exploración general de los diabéticos en consultas }\end{array}$ & $\begin{array}{r}\text { CON } \\
\text { MATERIAL }\end{array}$ & $\begin{array}{r}\text { SIN } \\
\text { MATERIAL }\end{array}$ & $\begin{array}{r}\text { TOTAL } \\
\text { CONSULTAS }\end{array}$ \\
\hline Consultas de medicina de familia: & $\mathbf{1 3 8}$ & $\mathbf{8 4 . 7}$ & 20 & $\mathbf{6 9}$ & $\mathbf{1 5 8}$ \\
\hline Consultas de pediatría: & 25 & $\mathbf{1 5 . 4}$ & 9 & $\mathbf{3 1}$ & $\mathbf{3 4}$ \\
\hline Total de consultas visitadas: & $\mathbf{1 6 3}$ & $100 \%$ & $\mathbf{2 9}$ & $100 \%$ & $\mathbf{1 9 2}$ \\
\hline
\end{tabular}

\section{Tabla 1}


En el 54\% de las historias clínicas consta la exploración anual de pies, y en el 74\% de ellas se ha realizado categorización del riesgo.

En centros hospitalarios se han evaluado 18 consultas, de las cuales el 78\% disponen de monofilamentos y el 18\% de esfigmomanómetro, estando calibrados el 72\% .Disponen de material mínimo para la exploración general de los diabéticos el 50\%, y de material para la exploración del pié diabetico el 93\%. En las historias clínicas muestreadas, consta la realización de la exploración anual de pies en el $67 \%$ y está categorizado el riesgo en el $38 \%$ de ellas.
La evaluación de la impartición de educación diabetológica muestra que existen programas estructurados en el 100\% de los centros de salud (Tabla 3) y de los centros hospitalarios (Tabla 4).Existen instalaciones y materiales para las sesiones educativas en el $100 \%$ de los centros tanto de Atención Primaria como hospitalarios y está registrada dicha actividad en la historia clínica en el 10\% de las de centros de salud y en el $28 \%$ de las de centros hospitalarios.

Comparando los datos obtenidos en Málaga con los de Andalucía en su conjunto, se observa que en Málaga el porcentaje de consultas de

\begin{tabular}{|c|c|c|c|c|c|}
\hline $\begin{array}{l}\text { Disponibilidad de material mínimo para la } \\
\text { exploración de los pies de los diabéticos en consultas }\end{array}$ & $\begin{array}{r}\text { CON } \\
\text { MATERIAL }\end{array}$ & $\%$ & $\begin{array}{r}\text { SIN } \\
\text { MATERIAL }\end{array}$ & $\%$ & $\begin{array}{r}\text { TOTAL } \\
\text { CONSULTAS }\end{array}$ \\
\hline Consultas de medicina de familia: & 66 & 93 & 92 & 76 & 158 \\
\hline Consultas de pediatría: & 5 & 7 & 29 & 24 & 34 \\
\hline Total de consultas visitadas: & 71 & $100 \%$ & 121 & $100 \%$ & 192 \\
\hline
\end{tabular}

Tabla 2

\begin{tabular}{lrrrrrr}
$\begin{array}{l}\text { Existencia de programas estructurados de educación } \\
\text { diabetológica en consultas de Atención Primaria }\end{array}$ & INDIVIDUAL & $\%$ & GRUPAL & $\%$ & $\begin{array}{r}\text { TOTAL } \\
\text { CONSULTAS }\end{array}$ \\
\hline Consultas de medicina de familia: & $\mathbf{5 3}$ & $\mathbf{8 2}$ & 0 & $\mathbf{0}$ & $\mathbf{5 3}$ \\
\hline Consultas de pediatría: & $\mathbf{1 2}$ & $\mathbf{1 8}$ & 0 & $\mathbf{0}$ & $\mathbf{1 2}$ \\
\hline Total de consultas visitadas: & $\mathbf{6 5}$ & $100 \%$ & $\mathbf{0}$ & $100 \%$ & $\mathbf{6 5}$ \\
\hline
\end{tabular}

Tabla 3

\begin{tabular}{lrrrrr}
$\begin{array}{l}\text { Existencia de programas estructurados de educación } \\
\text { diabetológica en consultas hospitalarias }\end{array}$ & INDIVIDUAL & $\%$ & GRUPAL & $\%$ & $\begin{array}{r}\text { TOTAL } \\
\text { CONSULTAS }\end{array}$ \\
\hline $\begin{array}{l}\text { Consultas de Endocrinología/ MI/ Diabetes/ Hospital de } \\
\text { Día*: }\end{array}$ & $\mathbf{7}$ & $\mathbf{5 8}$ & 2 & $\mathbf{6 7}$ & $\mathbf{9}$ \\
\hline Consultas de pediatría: & $\mathbf{5}$ & $\mathbf{4 2}$ & 1 & $\mathbf{3 3}$ & $\mathbf{6}$ \\
\hline Total de consultas visitadas: & $\mathbf{1 2}$ & $100 \%$ & $\mathbf{3}$ & $100 \%$ & $\mathbf{1 5}$ \\
\hline
\end{tabular}

Tabla 4 
medicina de familia y enfermería que disponen de material mínimo de exploración general de los diabéticos es menor, $31.7 \%$ frente al 93\% de las revisadas en Andalucía

Esta diferencia es aún más acusada en las consultas de pediatría, ya que el porcentaje de consultas de pediatría que disponen de material mínimo es del 84\% para Andalucía y del 15.4 para Málaga.

En cambio el porcentaje de consultas de primaria que disponen de esfigmomanómetro y monofilamentos es algo mayor en Málaga con respecto a las cifras globales de Andalucía.

En cuanto a la anotación en la historia clínica de la exploración anual de los pies, es mayor en Málaga frente a Andalucía. Sin embargo, la categorización del riesgo es superior en Andalucía ( 88.5\%).

La existencia de programas de educación diabetológica en primaria es menor en Málaga.

Con respecto a los datos obtenidos en los hospitales, si bien las consultas que están dotadas de monofilamentos son mayores en Málaga, llama la atención la escasa dotación de esfigmomanómetros en las consultas de Málaga, siendo también menor el porcentaje de centros con material mínimo para la exploración, que en Andalucía es del 84\%.

La anotación en la historia clínica de la exploración anual de pies es mayor en Málaga, pero no así en la categorización del riesgo, ya que el porcentaje de esta anotación es del $79 \%$ para Andalucía.

La existencia de programas estructurados de educación diabetológica es superior en los hospitales de Málaga con respecto a los hospitales andaluces, siendo similar el porcentaje de historias clínicas en donde consta la anotación.

Con respecto a la evaluación de las normas de calidad de los procesos Diabetes I y II, en Centros de Salud han sido han evaluadas la determinación de HbA1c (con carácter anual), valores de ese parámetro por debajo del $8 \%$, pacientes con estudio de fondo de ojo anual, y estudio de pies anual, mientras que en centros hospitalarios se han evaluado la realización de estudio de fondo de ojo anual, planificación de la gestación en mujeres diabéticas, y pacientes tratados en oftalmología por retinopatía.

Los resultados muestran que en Centros de Salud se alcanza un alto grado de cumplimiento en lo que respecta a la determinación de HbA1c $(70 \%)$, siendo menor el grado de cumplimiento de la realización de fondo de ojo anual registrado $(53 \%)$ y exploración de pies $(36 \%)$. El porcentaje de pacientes con $\mathrm{HbA} 1 \mathrm{c}$ con valores por debajo del $8 \%$ cuya determinación consta en la historia clínica es del $75 \%$.

En los hospitales evaluados, el porcentaje de pacientes en donde se ha planificado la gestación es del 49\%. Los pacientes tratados en oftalmología han sido tratados con laserterapia en un $53 \%$ y con cirugía el $46.9 \%$. Tabla 2

\section{DISCUSIÓN}

Los resultados obtenidos muestran que en Atención Primaria la disponibilidad de material mínimo de exploración general del diabético puede no ser suficiente, así como la dotación de esfigmomanómetros, aunque habría que incidir en la calibración de los mismos. La dotación de material mínimo para la exploración de pies es aceptable, ya que lo poseen el $93 \%$ de las consultas de medicina de familia, así como la dotación de monofilamentos para la exploración de la sensibilidad del pié.

En los hospitales revisados, consideramos aceptable el número de centros que disponen de material mínimo para la exploración de pies, pero habría que incidir en aumentar el material para la exploración general del diabético. En cuanto a la educación diabetológica, la totalidad de los centros de salud y hospitales realizan programas estructurados, en la mayor parte de los casos de forma individual, y cuentan con materiales e instalaciones suficientes en los centros de salud. El registro de esta actividad en la historia clínica es escaso tanto en Atención Primaria como en los centros hospitalarios.

Sería conveniente contemplar la sistematización en las exploraciones de los pies en el ámbito de la Atención Primaria, a las personas que les han tipificado pié de riesgo, haciéndolo como mínimo cada seis meses. De esta manera se conseguiría incrementar la prevención de la aparición del pie diabético, constituyendo así la base para la atención integral que se merece.

Se contempla en el futuro un incremento de la prevalencia de la DM, siendo posible que pueda alcanzar proporciones epidémicas. Ello 
generaría un aumento indudable de las complicaciones asociadas a la diabetes $\mathrm{y}$, en concreto, de un problema tan complejo como es el pie diabético, por tanto, sería de gran utilidad la creación en el ámbito hospitalario de una unidad específica del pié diabético que implicara a Servicios de forma directa como Endocrinología y Nutrición, Cirugía Vascular, Cirugía Ortopédica y Traumatología, Fisioterapia, que incluyera además del personal médico y de enfermería específico al fisioterapeuta y al podólogo como experto en la valoración y diseño de las descargas soportes plantares, creándose un circuito específico entre Atención Primaria y Especializada para este tipo de pacientes con historial clínico compartido.

Sería muy interesante la realización de estudios coste-efectividad de las diferentes intervenciones posibles que permitan un debate más transparente sobre esta cuestión, valorando por ejemplo tiempo promedio de llegar a amputación quirúrgica en

pacientes a los que se ha sometido a un adecuado tratamiento ortopodológico de descargas plantares y a los que no.

\section{BIBLIOGRAFÍA}

1. Ministerio de Sanidad y Consumo. Estrategia en Diabetes del Sistema Nacional de Salud. Sanidad 2007. Ministerio de Sanidad y Consumo.2007.85p.

2. Consejería de Salud. Junta de Andalucía. Plan Integral de diabetes:2003-2007. Sevilla. Consejería de Salud. 2002.224p.

3. Torrecillas A, Ríos C, Aguilar A, Ruiz MJ, Corchado Y, Fernández I. Prevalencia de diabetes tratada con fármacos en Andalucía. Evolución de la prescripción de antidiabéticos orales e insulina entre 1994 y 1998. Atención Primaria. 1999, 24 Suppl 2:319.

4. Ruiz-Ramos M, Escolar-Pujolar A, Mayoral-Sanchez E, Corral-SanLaureano F. La diabetes mellitus en España: Mortalidad,Prevalencia, Incidencia, Costes económicos y desigualdades. Gac Sanit.2006; Suppl 1:15-24.

5. Ester Vilá B, Rocha Blasco B,Sanz de los Arcos B, Padrós Sánchez C, Tratamiento de las úlceras neuropáticas con descargas. Revista española de podología. 2008; 19: 144-153.

6. Consejería de Salud. Junta de Andalucía. Diabetes mellitus tipo II: proceso asistencial integrado. Sevilla. Consejería de Salud 2002. 224 p.

7. Delgado Díaz DC, Herrera E, Camargo Lemos DM. La prueba de los monofilamentos: una alternativa para la detección oportuna del riesgo de pie diabético. Salus-UIS.2004;36:32-39. 